\title{
Life prediction for aircraft structure based on Bayesian inference: towards a digital twin ecosystem
}

\author{
Teng Wang ${ }^{1}$, Zheng Liu $^{2}$, Min Liao $^{3}$ and Nezih Mrad ${ }^{4}$ \\ ${ }^{1,2}$ Teng Wang, Zheng Liu, are with the school of Engineering, the University of British Columbia Okanagan Campus, \\ Kelowna, British Columbia VIV 1V7, Canada City \\ teng.wang@ubc.ca \\ zheng.liu@ubc.ca \\ ${ }^{3}$ Min Liao is with the National Research Council Canada, Aerospace Research Centre, \\ Ottawa, K1A 0R6, Canada \\ min.liao@nrc-cnrc.gc.ca \\ ${ }^{4}$ Nezih Mrad is with the Department of National Defence, Government of Canada, \\ Ottawa, K1A 0K2, Canada \\ nezih.mrad@forces.gc.ca
}

\begin{abstract}
Nowadays, the concept of "digital twin" has received great attention from both academia and industry. However, few methodological solutions have been reported in the existing studies. This paper presents a life prediction method for aircraft structure, and illustrates how this method can be embedded into a "digital twin" framework. This method can fuse heterogeneous information acquired from inspected physic entity, finite element software, historical database and predictive model, giving an accurate and real-time prediction of the remaining useful life (RUL) for aircraft structure. In the operation of this method, the degradation behavior of inspected structure is observed in an online manner. Historical record document is used for generating prior knowledge. The external load condition is fed into finite element software for calculating the stress intensity factor. The well-known paris law is adopted as the predictive model. Finally, the Bayesian inference is used to integrate the information and predict the future degradation of the inspected structure. Theoretical deviation and experiment on a public database demonstrate the effectiveness of this method, facilitating the implementation of "digital twin" in real-world scenario.
\end{abstract}

\section{INTRODUCTION}

The expression "digital twin" has become a generic term used across disciplines to mean a digital copy of a physical

\footnotetext{
Teng Wang et al. This is an open-access article distributed under the terms of the Creative Commons Attribution 3.0 United States License, which permits unrestricted use, distribution, and reproduction in any medium, provided the original author and source are credited.
}

entity. According to the United States Department of Defense (DoD), "digital twin" is defined as, "An integrated multiphysics, multiscale, probabilistic simulation of an as-built system, enabled by digital thread, that uses the best available models, sensor information, and input data to mirror and predict activities/performance over the life of its corresponding physical twin."

Modern aerospace industry is migrating from reactive maintenance to proactive and predictive maintenance for increasing platform operational availability and efficiency (Liu, Meyendorf, \& Mrad, 2018). In this regard, "Digital Twin" is a promising technique that improves predictive accuracy and minimizes life-cycle cost. As forecasted in many studies (Liao, Renaud, \& Bombardier, 2020; Glaessgen \& Stargel, 2012), this conception has the great potential to bring innovative and revolutionary changes for the aerospace engineering.

In the concept level, many studies have been carried to assess the adaptability of "digital twin" in aerospace engineering. The typical research works on life prediction for aircraft structure are as follows. The U. S. Air Force has developed an individual aircraft tracking (IAT) program to track the potential structural crack growth in the major airframe structural components of F-16 (Lee, Cho, \& Park, 2012). Then, this IAT program is investigated to realize the "digital twin" concept for fusing multiple heterogeneous sources of information (Bond, Underwood, Adams, \& Cummins, 2014). The National Research Council of Canada (NRC) currently demonstrates an aircraft digital twin framework using a CF188 Full-Scale component test (Renaud, Liao, \& Bombardier, 
2019), for potential application to Royal Canadian Air Force (RCAF) fleets. This demonstration highlights the benefits of Bayesian updating for realizing a "digital twin" model.

Besides, some research efforts focus on studying potentially available methodologies towards digital twin data. For example, Seshadri et al. (Seshadri \& Krishnamurthy, 2017) studied damage size and location estimation of aircraft structure using the digital twin concept, where the software, Abaqus/CAE, Matlab GA optimization routine, Python scripting language were adopted for numerical simulation. $\mathrm{Li}$ et al. (Li, Mahadevan, Ling, Choze, \& Wang, 2017), adopted dynamic Bayesian network for aircraft wing health monitoring. This method integrates the test data, mathematical models and expert opinions for aircraft wing crack growth prediction, and is validated using a numerical simulation example. Millwater et al. (Millwater, Ocampo, \& Crosby, 2019) reviewed probabilistic methods with potentials for digital twin implementation and discussed the future challenges for "digital twin" modeling.

To further facilitate the implementation of "digital twin" in real-world scenario, this paper presents a life prediction method for aircraft structure based on Bayesian inference. The main contributions of this paper lie in: (1) we present a method that can fuse heterogeneous information acquired from inspected physical entity, finite element software, historical database and predictive model, giving an accurate and real-time prediction of remaining useful life (RUL) for aircraft structure. (2) we illustrate how this method can be embedded into a digital twin framework, facilitating the implementation of "digital twin" in real-world scenario.

The rest of this paper is organized as follows. Section 2 gives an interpretation of the "digital twin" for aircraft structure. Section 3 describes how to realize this "digital twin" based on Bayesian inference. The experimental validation of this method is given in Section 4, followed by the conclusion drawn in Section 5.

\section{INTERPRETATION OF DIGITAL TWIN FOR AIRCRAFT STRUCTURE}

One of the key advantages of digital twin is that it can integrate multi-source, multi-scale information from physical entity in a real-time manner, creating a living model for predictive maintenance. As shown in Fig.1, the presented "digital twin" model comprises following input elements.

- Load condition: Load condition information describes the external loads (aerodynamic pressure and ground loads) that the aircraft structure experienced. The external load will be related to local stresses at details such as fastener holes, cutouts, and fillets. Then the fatigue crack can be evaluated and predicted in these local areas. It is worth mentioning that the development of damage

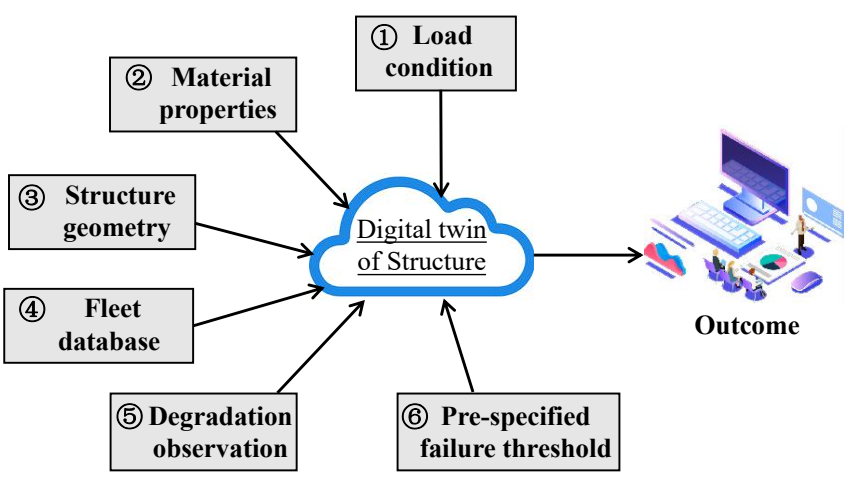

Figure 1. Diagram of the proposed method.

will affect the load at which inelastic deformations begin. Thus, the load condition should communicate with damage models ideally.

- Material property: Material property includes the parameters, such as Young's modulus, Poisson's ratio, constitutive model, which can be acquired from laboratory. The material property information will be fed into digital twin finite element model (FEM) to perform nonlinear analysis.

- Structure geometry: Structure geometry document describes the detailed dimension information of physical entity. This information helps to build a counterpart in digital FEM, which is necessary for identifying stress concentration, and calculating the related parameters for damage model.

- Fleet database: This database records the degradation history of the same batch of aircraft structures. After every physical aircraft flies, data about the flight will be downloaded from individual aircraft tracking (IAT) system. These historical information is used to parameterize the individual aircraft models from a probabilistic view. Specifically, various parameters related to the aircraft structure will be described in mean, standard deviation, distribution type, etc.

- Degradation observation: Degradation observation is the observed degradation behavior (such as crack propagation) of the inspected aircraft structures. New observation is obtained from the actual aircraft structure with a certain sampling interval. For example, the fatigue crack length can be measured using non-destructive testing (NDT) technology. Then the damage model can be updated accordingly.

- Pre-specified failure threshold: Failure threshold is specified by handbooks or expert, and defines the failure of aircraft structure when damage exceeds a certain level. By comparing the predicted degradation trend and prespecified failure threshold of the inspected aircraft struc- 
ture, we can assess the remaining useful life (RUL) during its service.

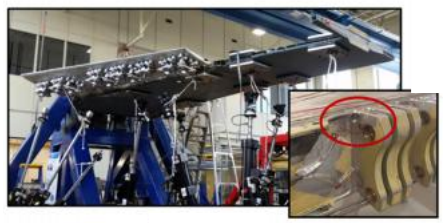

(a)

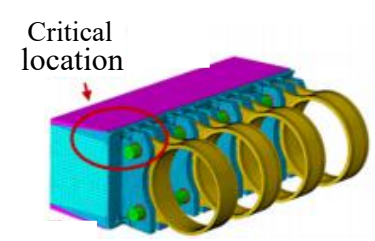

(b)
Figure 2. An illustrative example for CF-199 inboard leading edge flap (ILEF).

To further illustrate the implementation of "digital twin", we exhibit an example in Fig. 2 where the aircraft structure, CF199 inboard leading edge flap (ILEF) shown in Fig. 2(a), is modeled. First, the load condition can be acquired from the strain gauge located on the ILEF. Then, based on the material property and structure geometry, we can identify the critical location on ILEF which suffers from fatigue crack as shown in Fig. 2(b). The fleet database records the fatigue crack growth and load history of ILEF of other aircraft in the fleet. The degradation observation, i.e., the crack length at critical location, can be obtained by operator via NDT technology in maintenance activities. Then the presented method can output the prediction of the remaining useful life for the inspected ILEF. As a result, the real-world maintenance activities can be guided and optimized.

The next section describes how to integrate above information in the methodology level and output the RUL of the inspected aircraft structure.

\section{METHOD IMPLEMENTATION}

The life prediction method embedded in the "Digital Twin" framework can forecast the degradation of aircraft structure during the virtual flight. Generally, damage of aircraft structure includes fatigue crack, creep, fretting, wear, delamination, corrosion and oxidation (Tuegel, Ingraffea, Eason, \& Spottswood, 2011). Among them, the fatigue crack is the most common damage and thus is taken for studying in this paper. This paper focuses on predicting the crack growth tendency using Bayesian inference and giving the RUL for the inspected entity.

Fig. 3 illustrates the flowchart of the presented method which is based on the well-known Paris law (Paris \& Erdogan, 1963) as,

$$
\frac{d a}{d N}=C \Delta K^{m}
$$

where $a$ is the crack length, and $N$ is the number of cycles that

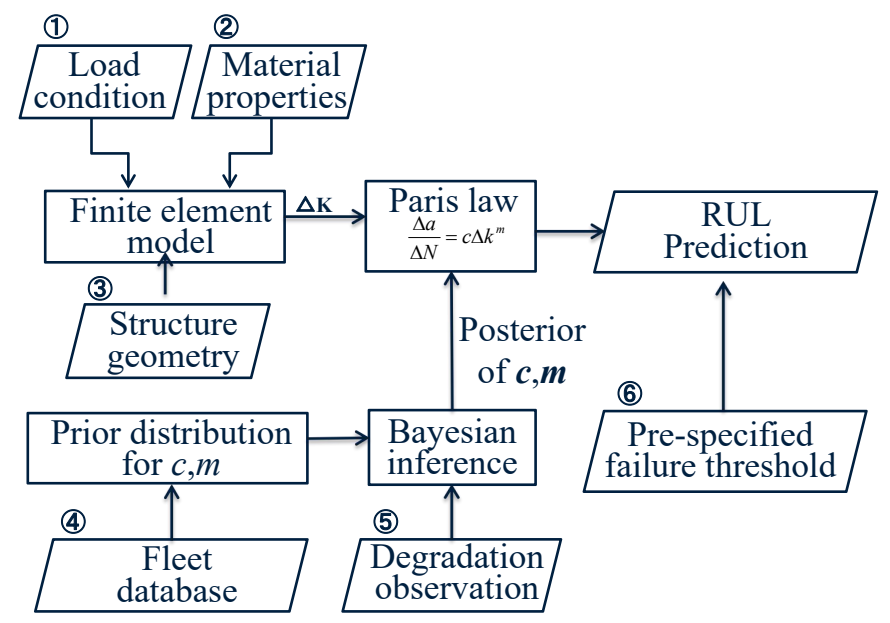

Figure 3. Presented method for RUL prediction for aircraft structure.

the structure experienced. The unknown parameters include $\Delta K, C$ and $m$.

Specifically, $\Delta K$ is the so-called stress intensity factor (SIF). It describes the stress field in the region near the crack tip. As shown in Fig. 3, by feeding the load condition, material property and structure geometry into finite element software, we can get the exact SIF at the crack tip. An illustrative example is given in Fig. 4 where the finite element software, ANSYS, is used for calculating the SIF under specific load condition.

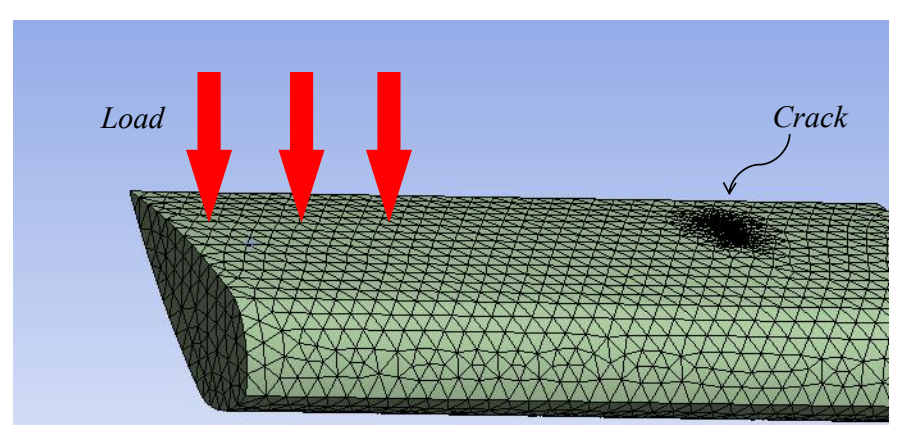

Figure 4. Crack simulation using ANSYS.

The parameter $C$ and $m$ are material constants for fatigue crack growth. Namely, for a specific structure, $C$ and $m$ are constants. For a same batch of aircraft structure, $C$ and $m$ are considered as statistically correlated random variables. Meanwhile, many studies (An, Choi, \& Kim, 2012; Ortiz \& Kiremidjian, 1988) point out that $(m, \log C)$ is assumed to obey a bivariate Gaussian distribution. In this paper, the bivariate Gaussian distribution of $(m, \log C)$ is acquired from the fleet database which records the fatigue growth trajectories of a set of structures served previously. Giving a set of crack growth trajectories, we can calculate the corresponding bivariate Gaussian distribution by the following steps, 
(1) Calculate the $\Delta K$ regarding different crack length and load using finite element software.

(2) Calculate the $d a / d N$ regarding different crack length using secant method (Clark \& Hudak, 1975).

(3) Apply the natural logarithm in the both sides of (1), we can get,

$$
\log \frac{d a}{d N}=\log C+m \log \Delta K
$$

Thus, by fitting the transferred crack growth trajectories, we can get a set of $(m, \log C)$ regarding trajectories.

(4) Estimate the parameters of bivariate Gaussian distribution by fitting $(m, \log C)$.

So far, we have introduced how to got prior distribution of $(m, \log C)$ using historical record document.

Then, given a specific structure, we can observe the crack length $a_{1}, \ldots, a_{t}$ at inspected time $t$. Based on Bayesian inference, the posterior distribution of $(m, \log C)$ is calculated as,

$$
\begin{aligned}
& P\{(m, \log C) \mid\left.a_{1}, \ldots, a_{t}\right\}= \\
& L\left\{a_{1}, \ldots, a_{t} \mid(m, \log C)\right\} * P\{(m, \log C)\},
\end{aligned}
$$

where $P\{(m, \log C)\}$ is prior distribution of $(m, \log C)$ that is assumed to follow the bivariate Gaussian distribution $N\left(\mu_{1}, \mu_{2}, \sigma_{1}^{2}, \sigma_{2}^{2}, \rho\right)$ as,

$P\{(m, \log C)\}=\frac{1}{(\sqrt{2 \pi})^{2} \sqrt{|C|}} \exp \left[-\frac{1}{2}(X-\mu)^{T} C^{-1}(X-\mu)\right]$

where

$$
X=\left[\begin{array}{c}
m \\
\log C
\end{array}\right], \mu=\left[\begin{array}{l}
\mu_{1} \\
\mu_{2}
\end{array}\right], C=\left[\begin{array}{cc}
\sigma_{1}^{2} & \rho \sigma_{1} \sigma_{2} \\
\rho \sigma_{1} \sigma_{2} & \sigma_{2}^{2}
\end{array}\right]
$$

$L\left\{a_{1}, \ldots, a_{t} \mid(m, \log C)\right\}$ is the likelihood function that is calculated as,

$$
\begin{aligned}
& L\left\{a_{1}, \ldots, a_{t} \mid(m, \log C)\right\}= \\
& \Pi_{k=1}^{t} \frac{1}{\sqrt{2 \pi} \sigma} \exp \left[-\frac{1}{2 \sigma^{2}}\left(\left.\frac{d a_{k}}{d N}\right|_{\text {esti }}-\left.\frac{d a_{k}}{d N}\right|_{a c t}\right)^{2}\right]
\end{aligned}
$$

where $\left.\frac{d a_{k}}{d N}\right|_{\text {esti }}$ and $\left.\frac{d a_{k}}{d N}\right|_{a c t}$ is the estimated crack growth speed and actual crack growth speed at crack length $a_{k}$ respectively. $\sigma$ is the standard deviation of prediction error.
Till now, we have defined the prior distribution and likelihood function, thus the posterior distribution $P\left\{(m, \log C) \mid a_{1}, \ldots, a_{t}\right\}$ can be calculated. It is worth mentioning that most of time (3) only has conceptual solution, which can not be obtained analytically. Therefore, Monte Carlo method should be adopted to approximate $P\left\{(m, \log C) \mid a_{1}, \ldots, a_{t}\right\}$ numerically. In this paper, a stateof-the-art Monte Carlo method, No-U-Turn Sampler (NUTS), is adopted for this purpose (Hoffman \& Gelman, 2014). More details about Monte Carlo method can be found in (Andrieu, De Freitas, Doucet, \& Jordan, 2003).

In summarize, we have got the $\Delta K$ using the finite element software, and $C, m$ using Bayesian inference. As a result, the crack length speed $d a / d N$ can be predicted from (1).

Unfortunately, Eq. (1) can not be integrated in a closed form. Hence, the Newton-Raphson method is adopted for predicted the crack length $a$ according to $d a / d N$. Let us denote the incremental loading cycles be $\Delta N$ and crack length after $i \Delta N$ load cycles is $a(i \Delta N)$. Then the crack length after $(i+1) \Delta N$ load cycles can be calculated as,

$$
a((i+1) \Delta N)=a(i \Delta N)+(d a(i \Delta N) / d N) \Delta N
$$

Hence, we can calculate the crack length $a$ after a certain amount of load cycles. By comparing with a pre-specified failure threshold, we can finally estimate RUL of the inspected aircraft structure.

\section{EXPERIMENT}

The proposed method is validated using fatigue crack growth data reported in (Virkler, Hillberry, \& Goel, 1979). The tested specimens were 0.10 inch specimens of 2024-T3 aluminum alloy as shown in Fig. 5. A stress raiser was machined in the center of this specimen. The reason for choosing the 2024T351 aluminum alloy is owing to its popularity in use for the aging aircraft made in the seventies. The tests were run at room temperature in a random order on a 20 Kip electrohydraulic closed loop system operated in load control with loads controlled within 0.2 percent. A sinusoidal signal with a frequency of $20 \mathrm{~Hz}$ was used as the input for the alternating portion of the load. More details about this experiment can be found in (Virkler et al., 1979).

In this experiment 68 specimens were tested, their crack growth trajectories are given in Fig. 6. In the following, specimen 1 and specimen 68 are selected for inspection respectively as they have the shortest and the longest failure time.

\subsection{Case 1}

In Case 1, specimen 1 is taken for testing. The specimens 2-68 are regarded as the previously served structure whose 


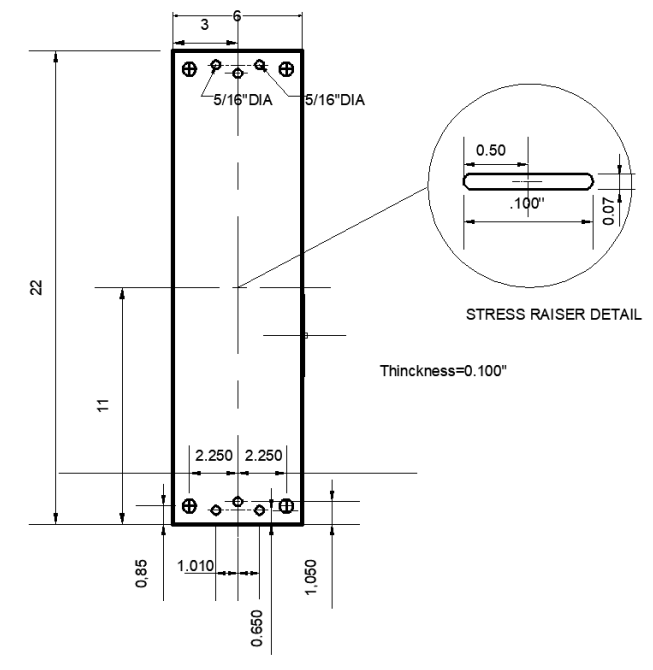

Figure 5. The schematic of the tested specimen.

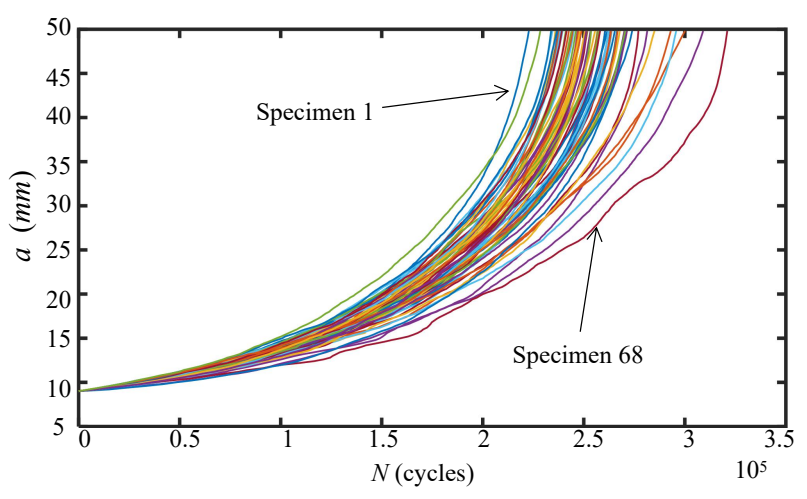

Figure 6 . The crack growth trajectory of 68 specimens.

degradation trajectories are recorded in the fleet database. Thus specimens 2-68 are used to calculate the prior distribution of $(m, \log C)$. Fig. 7 shows the calculated bivariate Gaussian distribution $N_{\text {case } 1}\left(\mu_{1}, \mu_{2}, \sigma_{1}^{2}, \sigma_{2}^{2}, \rho\right)$ where $\mu_{1}=$ $2.8719, \mu_{2}=-16.2242, \sigma_{1}^{2}=0.0273, \sigma_{2}^{2}=0.1608, \rho=$ -0.9889 . The calculated distribution is exhibited in Fig. 7 where each scatter denotes one pair of $(m, \log C)$ corresponding to a specimen. It is worth mentioning that even physically identical components made of the same type of material could demonstrate different fatigue behavior. From Fig. 7, we can see that in addition to a few outliers, the twodimensional Gaussian distribution can generally fit these scatters.

Then, using the Bayesian inference described in Section 3, we can obtain the value of $C, m$ based on real-time degradation observation.

Meanwhile the $\Delta K$ with respect to different crack length $a$ is exhibited in Fig. 8, where we can see $\Delta K$ increases as the crack grows. As $\Delta K, C, m$ are all available, the correspond- ing $d a / d N$ at specific crack length can be calculated using (1). Finally, we can predict future crack length using (7).

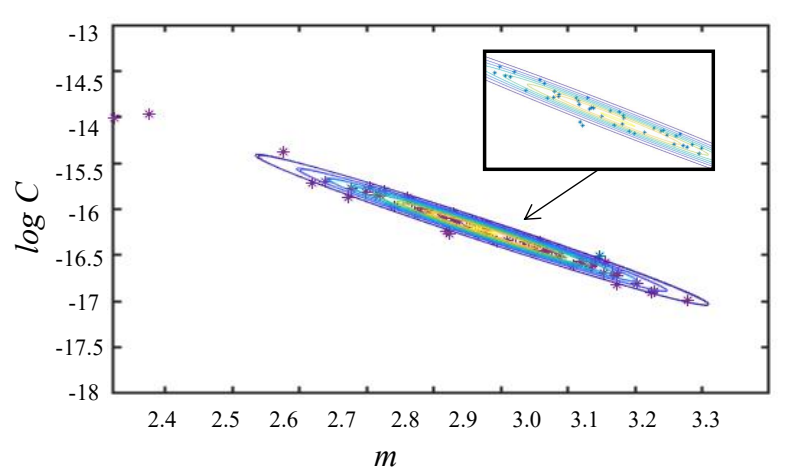

Figure 7. The prior distribution of $(m, \log C)$ calculated in Case 1.

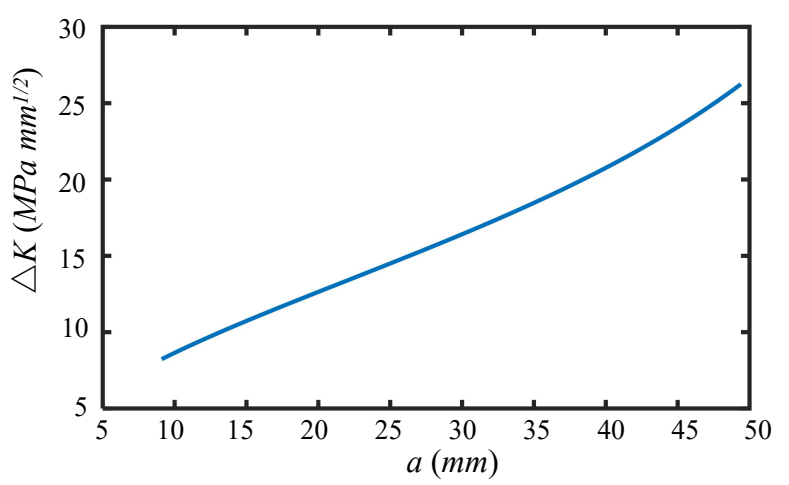

Figure 8. The value of $\Delta K$ with respect to different crack length $a$.

Then, we define the failure threshold as $49.8 \mathrm{~mm}$. Namely, when crack length of inspected specimen exceeds $49.8 \mathrm{~mm}$, this specimen will be regarded as failure. Since the lifetime of aircraft structure depends on the load that it experienced, in this study, we use load cycle to replace the time for convenience. For example, $R U L=100000$ means the inspected structure can still bear 100000 load cycles before its failure.

Fig. 9 presents the RUL estimation result of specimen 1 at different time. It can be seen that the predicted RUL is close to the actual one, which shows the effectiveness of the presented method for RUL prediction.

\subsection{Case 2}

Specimen 68 is taken for testing in Case 2. The specimens 1-67 are regarded as the previously served structures that are recorded in the fleet database. Thus specimens 167 are used to calculate the prior distribution of $(m, \log C)$. Fig. 10 shows the calculated bivariate Gaussian distribution $N_{\text {case } 2}\left(\mu_{1}, \mu_{2}, \sigma_{1}^{2}, \sigma_{2}^{2}, \rho\right)$ where $\mu_{1}=2.8752, \mu_{2}=$ $-16.2277, \sigma_{1}^{2}=0.0277, \sigma_{2}^{2}=0.1620, \rho=-0.9895$. We 


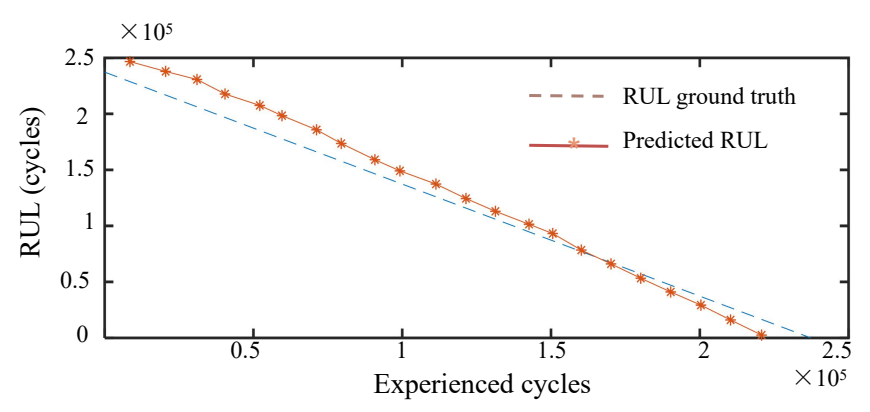

Figure 9. The RUL prediction result in Case 1.

can see that $N_{\text {case } 1}$ and $N_{\text {case } 2}$, are also very close, as they all denote the prior knowledge of a same batch of specimen.

On the other hand, relationship between the $\Delta K$ and crack length $a$ is as same as that exhibited in Fig. 8. Without going into details, we present the RUL prediction result of case 2 in Fig. 11. We can see that the prediction error at the initial degradation stage is relatively large, and when more degradation observations are available, the prediction result gets closer to the actual RUL. Meanwhile, it is worth mentioning that the prediction results in Case 2 are always less than the ground truth. It is because specimen 68 has the lowest crack growth speed which can be seen in Fig. 6. Since in Bayesian inference, the prior distribution is calculated from specimen 1-67 which have higher crack growth speed than specimen 68. Thus, the crack growth speed of specimen 68 is always overestimated. As a result, the RUL of specimen 68 is always underestimated.

Overall, the presented method is also effective for the RUL prediction in Case 2.

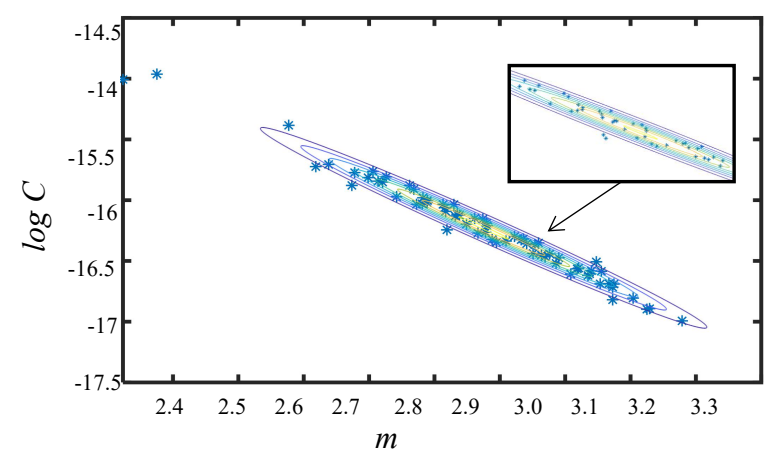

Figure 10. The prior distribution of $(m, \log C)$ calculated in Case 2.

\section{Conclusion}

This paper presented a life prediction method for aircraft structure, and illustrates how this method can be embedded into a "digital twin" framework. This method can fuse heterogeneous information including load condition, material prop-

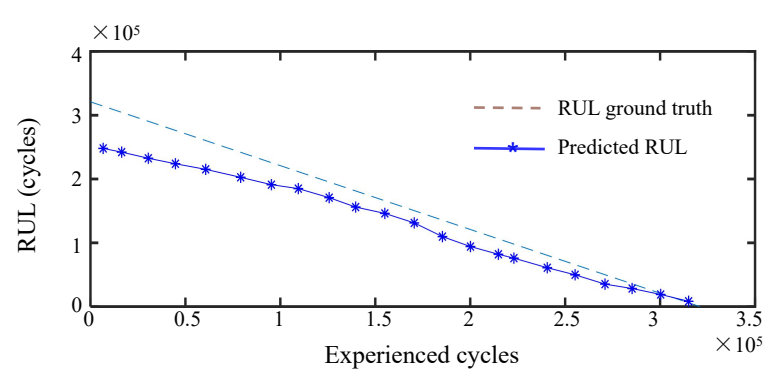

Figure 11. The RUL prediction result in Case 2.

erties, structure geometry, historical record document, degradation observation and pre-specified failure threshold. This method is based on the well-known Paris law. In its operation, the degradation behavior of the inspected structure is observed in an online manner. Fleet database is used for generating prior knowledge. The real-time load condition is used for calculating the stress intensity factor. Finally, the Bayesian theory is used to integrate these multi-sources of information and predict the remaining useful life (RUL) of the inspected structure.

Experiment on a crack propagation database shows that the presented method can give an accurate prediction of RUL for the aircraft structure. Thus, the presented method can facilitate the implementation of "Digital Twin" in real-world scenario.

\section{ACKNOWLEDgment}

This research is partially supported by the National Research Council of Canada through the CSTIP Grant (agreement CDTS-104).

\section{REFERENCES}

An, D., Choi, J.-H., \& Kim, N. H. (2012). Identification of correlated damage parameters under noise and bias using Bayesian inference. Structural Health Monitoring, 11(3), 293-303.

Andrieu, C., De Freitas, N., Doucet, A., \& Jordan, M. I. (2003). An introduction to MCMC for machine learning. Machine learning, 50(1-2), 5-43.

Bond, R., Underwood, S., Adams, D. E., \& Cummins, J. J. (2014). Structural health monitoring-based methodologies for managing uncertainty in aircraft structural life assessment. Structural Health Monitoring, 13(6), 621-628.

Clark, W., \& Hudak, S. (1975). Variability in fatigue crack growth rate testing. Journal of Testing and Evaluation, 3(6), 454-476.

Glaessgen, E., \& Stargel, D. (2012). The digital twin paradigm for future NASA and US air force vehicles. In 53rd aiaa/asme/asce/ahs/asc structures, 
structural dynamics and materials conference 20th aiaalasmelahs adaptive structures conference 14th aiaa (p. 1818-1832).

Hoffman, M. D., \& Gelman, A. (2014). The No-U-Turn sampler: adaptively setting path lengths in hamiltonian monte carlo. Journal of Machine Learning Research, 15(1), 1593-1623.

Lee, H., Cho, H., \& Park, S. (2012). Review of the f-16 individual aircraft tracking program. Journal of aircraft, 49(5), 1398-1405.

Li, C., Mahadevan, S., Ling, Y., Choze, S., \& Wang, L. (2017). Dynamic bayesian network for aircraft wing health monitoring digital twin. Aiaa Journal, 55(3), 930-941.

Liao, M., Renaud, G., \& Bombardier, Y. (2020). Airframe digital twin technology adaptability assessment and technology demonstration. Engineering Fracture Mechanics, 225, 106793.

Liu, Z., Meyendorf, N., \& Mrad, N. (2018). The role of data fusion in predictive maintenance using digital twin. In Aip conference proceedings (Vol. 1949, p. 020023).

Millwater, H., Ocampo, J., \& Crosby, N. (2019). Probabilistic methods for risk assessment of airframe digital twin structures. Engineering Fracture Mechanics, 221, 106674.

Ortiz, K., \& Kiremidjian, A. S. (1988). Stochastic modeling of fatigue crack growth. Engineering Fracture Mechanics, 29(3), 317-334.

Paris, P., \& Erdogan, F. (1963). A critical analysis of crack propagation laws. J Basic Eng ASME, 85(4), 528-533.

Renaud, G., Liao, M., \& Bombardier, Y. (2019). Demonstration of an airframe digital twin framework using a CF-188 full-scale component test. In International committee on aeronautical fatigue (pp. 176-186).

Seshadri, B. R., \& Krishnamurthy, T. (2017). Structural health management of damaged aircraft structures using digital twin concept. In 25th aiaa/ahs adaptive structures conference (p. 1675).

Tuegel, E. J., Ingraffea, A. R., Eason, T. G., \& Spottswood, S. M. (2011). Reengineering aircraft structural life prediction using a digital twin. International Journal of Aerospace Engineering, 2011.

Virkler, D. A., Hillberry, B., \& Goel, P. (1979). The statistical nature of fatigue crack propagation.

\section{BIOGRAPHIES}

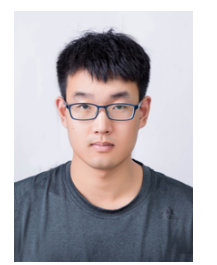

Teng Wang received his bachelor degree in mechanical engineering from Hefei University of Technology, Hefei, China, in 2016, and master degree in mechanical engineering from Shandong University, Jinan, China, in 2019. He is currently working toward the Ph.D. degree in School of Engineering, the University of British Columbia (Okanagan campus), Kelowna, Canada.
His research interest includes machinery condition monitoring, intelligent fault diagnostics, and remaining useful life prediction of machinery.

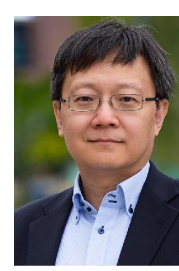

Zheng Liu (S'99-M'02-SM'06) received the Doctorate degree in engineering (earth resources) from Kyoto University, Kyoto, Japan, in 2000, and the Ph.D. degree (electrical engineering) from the University of Ottawa, Canada, in 2007. From 2000 to 2001, he was a Research Fellow with the Nanyang Technological University, Singapore. He then joined the National Research Council of Canada (Ottawa, Ontario) as a Governmental Laboratory Visiting Fellow nominated by NSERC in 2001. From 2002, he became a Research Officer associated two research institutes of NRC (Aerospace [IAR] \& Construction [IRC]). From 2012 to 2015, he worked as a full Professor with Toyota Technological Institute, Nagoya, Japan. He is now with the Faculty of Applied Science at the University of British Columbia Okanagan as an associate professor. His research interests include predictive maintenance, data/information fusion, computer/machine vision, machine learning, smart sensor and industrial IoT, and non-destructive inspection and evaluation. He is a senior member of IEEE and SPIE. He is co-chairing the IEEE Instrumentation and Measurement Society technical committee (TC-1). He holds a Professional Engineer license in both British Columbia and Ontario. Dr. Liu serves on the editorial boards for journals including IEEE Transactions on Instrumentation and Measurement, IEEE Transactions on Mechatronics, IEEE Journal of RFID, Information Fusion (Elsevier), Machine Vision and Applications (Springer), Canadian Journal of Electrical and Computer Engineering, Intelligent Industrial Systems (Springer), and IET/CAAI Transactions on Intelligence Technology. He also serves the Standards \& Interoperability Committee of the Research Data Canada.

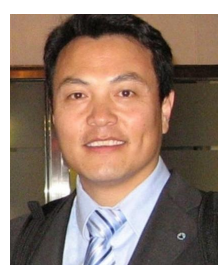

Min Liao received his $\mathrm{BE}$, MEng, $\mathrm{PhD}$ in Aerospace, Aeronautical and Astronautical Engineering, Northwestern Polytechnical University, Xi'an, China in 1986, 1989 and 1993 respectively.

In 1999, Dr. Liao joined in National Research Council of Canada (NRC), and now he is a Principal Research Officer, Group Leader of Structural Integrity in NRC Aerospace Portfolio. He also serves as a Thrust Lead (Life-Cycle Cost Reduction) of the NRC Air Defence System (ADS) program.

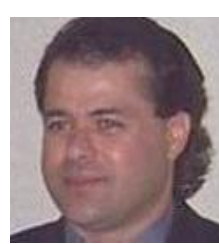

Nezih Mrad (SM'12) received the Ph.D. degree in engineering from Pennsylvania State University, University Park, in 1995, and two Masters degrees, in applied mathematics and in mechanical engineering, from the University of South Carolina, Columbia, in 1987 and 1988, respectively. He is currently a Defence Scientist with the Defence R\&D Canada (DRDC), Department of National Defence, Ottawa, ON, Canada. He is also an Adjunct Professor with Carleton University, Ottawa, and with the University of Sherbrooke, Sherbrooke, QC, Canada. He has intensive experience in advanced sensing, wireless sensors and sensor networks, energy harvesting, and RFID sensors. He conducts and manages R\&D activities in CBM, SHM, PHM, microand 
nanotechnologies, and autonomic logistics as they relate to aircraft for continued reduction of air platform life cycle costs, improved performance, increased safety and enhanced logistics support. He has authored or coauthored over 250 publications. Dr. Mrad is on the editorial board of several journals. He is the Lead Editor of several journals special issues. He is the Chair or a Co-Chair or a member of national, international and NATO committees, conferences, and workshops on issues ranging from structures and propulsion systems to nanotechnology. 